\title{
Produção de corante natural extraído de jambolão (Syzygium cumini)
}

\author{
Production of natural dye extracted from jambolan (Syzygium cumini) \\ Producción de colorante natural extraído del Syzygium cumini
}

Recebido: 02/01/2021 | Revisado: 07/02/2021 | Aceito: 08/02/2021 | Publicado: 15/02/2021

\author{
Bruna Ferreira Dias Freitas \\ ORCID: https://orcid.org/0000-0002-8848-4534 \\ Universidade Federal de Goiás, Brasil \\ E-mail: brunaferreira.mst@gmail.com \\ Gabriela Leite Magalhães \\ ORCID: https://orcid.org/0000-0001-7203-6430 \\ Universidade Federal de Goiás, Brasil \\ E-mail: gabriellaleite@ discente.ufg.br \\ Manoel Soares Soares Júnior \\ ORCID: https://orcid.org/0000-0001-8728-4592 \\ Universidade Federal de Goiás, Brasil \\ E-mail: manoel@ufg.br \\ Márcio Caliari \\ ORCID: https://orcid.org/0000-0002-0877-8250 \\ Universidade Federal de Goiás, Brasil \\ E-mail: macaliari@ufg.br
}

\begin{abstract}
Resumo
Dentre inúmeras frutas existentes no Brasil, o jambolão tem se destacado por ser rico em vários constituintes benéficos à saúde, como compostos fenólicos, e por apresentar propriedades antioxidantes e antimicrobianas. Ainda considerando os aspectos de relacionados à saúde, existe uma tendência dos consumidores para a utilização de corantes naturais em substituição os artificiais. Portanto, o presente trabalho teve como objetivo a avaliação química, física e propriedades tecnológicas da polpa de jambolão liofilizada e do corante natural proveniente do jambolão. Para obtenção do material liofilizado, a polpa foi seca em liofilizador industrial. Em seguida, o corante foi obtido por meio de extração utilizando como solvente água /etanol (proporção de 50:50), e posterior fixação utilizando como veículo farinha de arroz, seguido de secagem a $30^{\circ} \mathrm{C}$. A caracterização da polpa de jambolão liofilizada e corante natural em pó de jambolão foi realizada por meio da determinação das propriedades físico-químicas, composição proximal, compostos bioativos, índices de absorção de água (IAA), índice de solubilidade em água (ISA), índice de absorção de óleo (IAO), propriedade de pasta (RVA), estudo calorimétrico (DSC) e microscopia eletrônica de varredura. Os resultados demonstraram que o processo de extração e fixação do corante aumentaram a porcentagem de sequestro de radical livre de antioxidante $(6,4)$ e temperatura de pasta $\left(95,1^{\circ} \mathrm{C}\right)$, tratando-se de um corante termicamente estável quando comparado com a polpa liofilizada. Conclui-se que é possível a utilização polpa de jambolão liofilizada para produção de corante natural à base de jambolão e aplicação em alimentos.
\end{abstract}

Palavras-chave: Pigmento; Antocianinas; Compostos fenólicos; Capacidade antioxidante.

\begin{abstract}
Among the existing fruits in Brazil, jambolan has stood out for being rich in several beneficial components to health, such as phenolic compounds, and for having antioxidant and antimicrobial properties. Still considering aspects related to health, there is a tendency for consumers to use natural dyes instead of artificial ones. Therefore, the present work had as objective the chemical, physical and technological evaluation of the lyophilized jambolan pulp and the natural dye from the jambolan. To obtain the lyophilized pulp, the pulp was dried in an industrial freeze dryer. Then, the dye was added by means of extraction using water/ethanol (50:50 ratio), and later correction using rice flour as a vehicle, and drying at $30^{\circ} \mathrm{C}$. The characterization of lyophilized jambolan pulp and natural dye in jambolan powder was carried out by determining the physical-chemical properties, proximal composition, bioactive compounds, water absorption index (IAA), water solubility index (ISA), oil absorption index (IAO), the property of index paste (RVA), calorimetric study (DSC) and electronic research microscopy. The results showed that the dye extraction and correction process increased the percentage of free radical scavenging $(6.4)$ and paste temperature $\left(95.1{ }^{\circ} \mathrm{C}\right)$, being a thermally stable dye when compared to the lyophilized pulp. We conclude that it is possible to use lyophilized jambolan pulp for the production of natural jambolan dye and application in food.
\end{abstract}

Keywords: Pigment; Anthocyanins; Phenolic compounds; Antioxidant capacity. 


\begin{abstract}
Resumen
Entre las frutas existentes en Brasil, el Syzygium cumini (L) se ha destacado por ser rica en varios componentes beneficiosos para la salud, como los compuestos fenólicos, y por tener propiedades antioxidantes y antimicrobianas. Aún considerando aspectos relacionados con la salud, existe una tendencia de los consumidores a utilizar tintes naturales en lugar de artificiales. Por lo tanto, el presente trabajo tuvo como objetivo la evaluación química, física y tecnológica de la pulpa de Syzygium cumini (L) liofilizada y el tinte natural del Syzygium cumini (L). Para obtener la pulpa liofilizada, la pulpa se secó en un liofilizador industrial. Luego, se añadió el colorante mediante extracción con agua/etanol (relación 50:50), y posterior corrección con harina de arroz como vehículo, y secado a $30^{\circ} \mathrm{C}$. La caracterización de pulpa de Syzygium cumini (L) liofilizada y colorante natural en pulpa de Syzygium cumini (L) se llevó a cabo determinando las propiedades físico-químicas, composición proximal, compuestos bioactivos, índice de absorción de agua (IAA), índice de solubilidad en agua (ISA), índice de absorción de aceite (IAO), propiedad de la pasta índice (RVA), estudio calorimétrico (DSC). ) y microscopía de investigación electrónica. Los resultados mostraron que el proceso de extracción y corrección del tinte aumentó el porcentaje de captación de radicales libres (6.4) y la temperatura de la pasta $\left(95.1^{\circ} \mathrm{C}\right)$, siendo un tinte térmicamente estable en comparación con la pulpa liofilizada. Concluimos que es posible utilizar pulpa Syzygium cumini (L) liofilizada para la producción de tinte Syzygium cumini (L) natural y su aplicación en alimentos.

Palabras clave: Pigmento; Antocianinas; Compuestos fenólicos; Capacidad antioxidante.
\end{abstract}

\title{
1. Introdução
}

O jambolão pertence à família Myrtaceae de origem Asiática, especificamente da Índia. No Brasil encontra-se em diferentes regiões do país como um árvore ornamental, conhecida por diversos nomes, como jamelão, jambolão, azeitona preta, ameixa preta e amora silvestre indiana (Carvalho et al., 2016; Singh et al., 2016). Com formato de bagas elipsoidais, o jambolão possui comprimento de 3 a $5 \mathrm{~cm}$, polpa esbranquiçada ou rosada com uma semente localizada no centro da fruta, que dispõe de coloração verde e quando atinge a maturação apresenta uma coloração de roxa a preta (Singh; Singh, 2018).

No Brasil, a floração do jambolão inicia entre setembro a novembro e a maturação da fruta entre dezembro e fevereiro. São frutas não climatéricas, por isso é necessário deixar atingir o tempo de maturação completa para colheita (Sabino; De Brito; Da Silva Júnior, 2018), além disso, é uma fruta altamente perecível, devido à fragilidade da polpa e do epicarpo, provendo assim baixa proteção contra injúrias físicas e agentes infecciosos, deste modo não pode ser armazenada por longo tempo (Baraiya et al., 2015; Sabino et al., 2018).

A obtenção do corante natural é um meio de preservar as características da fruta, como os compostos bioativos e garantir a finalidade de realçar cor nos alimentos e bebidas, com potencial substituição do uso de corante sintético (Silva; Nunes; Gomes; Silva, 2018). Um dos compostos fenólicos que existe no jambolão são as antocianinas, é devida sua presença há iminente aplicação como pigmento natural, há também taninos hidrolisáveis, flavanonóis e flavonóis (Lestario et al., 2017; Oliveira et. al 2017). Além das propriedades antioxidantes, possui efeito antimicrobiano no crescimento de Salmonella thyphimurium, Shigella flexneri, Staphylococcus aureus e E. coli enterro toxigênica, principais patógenos responsáveis por doenças transmitidas por alimentos (Haque et al., 2017).

Diante do exposto, este trabalho teve como objetivo a avaliação química, física e propriedades tecnológicas da polpa de jambolão liofilizada e do corante natural proveniente do jambolão.

\section{Metodologia}

\section{Obtenção da matéria prima}

Os frutos de jambolão foram colhidos em Goiânia, Goiás, Brasil. A farinha de arroz (Maninho®, Grupo Comman) foi adquirida no comércio local da cidade de Goiânia, Goiás, Brasil. 


\section{Preparação do jambolão}

Os frutos foram selecionados, lavados em água corrente, sanitizados com solução de hipoclorito de sódio de 100 ppm por 10 minutos, enxaguados em água corrente e secos a $25{ }^{\circ} \mathrm{C}$ em estufa com circulação de ar (Tecnal, TE-394/4mp, São Paulo, Brasil). Posteriormente foram despolpados em despolpadora industrial (Bonina 0.25 df, Itabuna (BA), Brasil), armazenados em congeladores e em seguida liofilizados em liofilizador (Liobras, P 820, São Paulo, Brasil), para a realização de sua caracterização e posterior produção de corante.

\section{Extração do corante natural de jambolão}

Para a extração do corante de jambolão, utilizou-se a técnica aplicada em corante de urucum, utilizando como solvente água/etanol (na proporção de 50:50). A matéria-prima foi deixada por 16 horas à temperatura de $5^{\circ} \mathrm{C}$ em contato com os solventes. Em seguida, a solução foi filtrada em tecido organza. Para remoção dos resíduos o extrato foi centrifugado por 10 minutos a temperatura ambiente. Após a centrifugação o extrato foi concentrado a $35-38{ }^{\circ} \mathrm{C}$ em evaporador rotativo até redução de $50 \%$ do volume inicial. Para a obtenção do corante natural em pó o concentrado foi misturado (proporção de 100g/50 L) com farinha de arroz e submetido á secagem em estufa de bandejas (Tecnal, TE-394/4mp, São Paulo, Brasil), em escala piloto, com circulação forçada de ar, à temperatura média de $30^{\circ} \mathrm{C}$ por 8 horas (Prentice-Hernandez e Rusig, 1992).

\section{Composição proximal da polpa de jambolão e corante natural}

Para a caracterização do teor de umidade e lipídeos totais das amostras utilizou-se a metodologia $n^{\circ} 932.12$ proposta por AOAC (2006). A proteína bruta foi determinada pelo método de Kjeldahl, e a quantidade de nitrogênio conforme procedimento da AOAC (1995). O resíduo mineral fixo (cinzas) foi determinado utilizando o método oficial 940.26 da AOAC (2006). A determinação do teor de fibra alimentar total foi realizada tendo por base o método AOAC 985.29 (enzimáticogravimétrico). O teor de carboidratos foi calculado pelo método de diferença, subtraindo-se de cem os valores de umidade, extrato etéreo, proteína, fibra e cinzas (Sniffen et al., 1992).

\section{Análises físico-químicas}

Acidez titulável, pH, sólidos solúveis foram determinados segundo a AOAC (2010).

\section{Compostos bioativos}

Os compostos fenólicos totais foram determinados pelo método proposto por Larrauri, Rupérez e Saura-Calixto (1997) e as antocianinas monoméricas totais pelo método colorimétrico por diferencial de pH (Giusti E Wrolstad, 2003).

\section{Atividade antioxidante}

Para a medida da atividade antioxidante foi utilizada a metodologia descrita por Lopes-Lutz et al. (2008). A atividade antioxidante (\%) foi calculada através da Equação 1:

$$
\% A A=\left(\frac{\left(A_{\text {controle }}-A_{\text {amostra }}\right)}{A_{\text {controle }}}\right) \times 100
$$




\section{Características tecnológicas da polpa de jambolão e corante natural}

Os parâmetros colorimétricos $\left(L^{*}, a^{*}, b^{*}, c^{*}\right.$ e H) foram determinados utilizando colorímetro (Branking Meter minolta, BC-10, Ramsey, USA), de acordo com Oliveira (2012).

Os índices de absorção em água (IAA), solubilidade (ISA) e absorção em óleo (IAO) foram determinados segundo metodologia descrita por Anderson et al. (1969).

Para a determinação da atividade de água (Aw), utilizou-se um medidor (AqcuaLab, CX-2, Washington, EUA).

\section{Propriedades térmicas e de pasta}

As propriedades térmicas foram obtidas através de um calorímetro exploratório diferencial (TA Instruments, Q20 DSC, New Castle, EUA) segundo AACC (2000). As curvas de propriedade de pasta foram obtidas por meio de um viscosímetro (RVA - Perten Instruments, 4500, Hägersten Suécia) de acordo com método proposto pela AACC (2000).

\section{Microscopia eletrônica de varredura (MEV)}

As amostras foram caracterizadas do ponto de vista morfológico por microscopia eletrônica de varredura (MEV) pela metodologia proposta por Kestemnbach (1994).

\section{Análise estatística}

Os resultados das análises de todo o experimento foram avaliados através da exploração dos resultados experimentais realizado com quatro repetições em triplicata, totalizando doze unidades experimentais. Utilizou-se na pesquisa metodologia quantitativa, onde os resultados numéricos foram expressos em média e o desvio padrão das amostras (Pereira et al. 2018).

\section{Resultados e Discussão}

\section{Composição proximal e propriedades físico-químicas da polpa de jambolão e corante natural}

Os valores médios encontrados para os teores de umidade, cinzas, proteínas e lipídeos da polpa de jambolão liofilizada (PJAM) (Tabela 1), de acordo com Neacsu et al. (2015), o teor de carboidratos, fibras e a presença de fitoquímicos são essenciais para garantir uma melhor reformulação do produto desejado, e normalmente as farinhas provenientes de frutas possuem baixo teor de lipídeo.

Tavares et al. (2017) encontrou para umidade na polpa de jambolão liofilizada um valor de 5,14\%, teor inferior ao reportado neste estudo. Brito et al. (2017) estudaram a composição proximal da polpa de jambolão e relataram teor de umidade $(87,20 \%)$, proteína $(0,85 \%)$, lipídeos $(0,49 \%)$, cinzas $(0,23 \%)$ e carboidratos de $11,40 \%$. A umidade apresentou valor $90 \%$ maior que o encontrado nesse trabalho, fato já esperado, pois a determinação desse trabalho foi em polpa liofilizada. 
Tabela 1 - Médias e desvio padrão da composição proximal da polpa de jambolão liofilizada (PJAM) e do corante natural de jambolão (CJAM).

\begin{tabular}{ccc}
\hline Parâmetros & PJAM $^{\mathbf{1}}$ & CJAM $^{\mathbf{1}}$ \\
\hline Umidade $^{2}$ & $9,48 \pm 0,26$ & $9,98 \pm 0,11$ \\
Cinzas $^{2}$ & $3,39 \pm 0,28$ & $0,79 \pm 0,36$ \\
Lipídeos $^{2}$ & $0,35 \pm 0,00$ & $0,64 \pm 0,07$ \\
Proteínas $^{2}$ & $0,34 \pm 0,00$ & $0,63 \pm 0,01$ \\
Fibras Alimentar totais $^{2}$ & $28,6 \pm 0,06$ & $9,84 \pm 0,09$ \\
Carboidratos Totais $^{2}$ & $57,8 \pm 0,22$ & $78,1 \pm 0,25$ \\
\hline
\end{tabular}

${ }^{1}$ Valores expressos em médias seguidas pelos desvios-padrão

${ }^{2}{\mathrm{~g} 100 \mathrm{~g}^{-1}}^{2}$

Fonte: Autores.

Shelke et al. (2020), obteve um teor de lipídeo (0,35\%) similar ao encontrado neste estudo para a polpa de jambolão liofilizada, de acordo com a Tabela 1 o valor encontrado para CJAM foi 1,82 vezes maior, isto provavelmente ocorreu devido ao teor de lipídeos presente na farinha de arroz (0,46 g 100g-1) utilizada na produção do corante em pó.

Observa-se um baixo teor de proteínas na PJAM e um valor 1,85 vezes maior para o CJAM (Tabela 1). Este acréscimo se relaciona ao teor de proteínas informado no rótulo da farinha de arroz adicionada $(3,0 \mathrm{~g})$. Os resultados deste trabalho foram inferiores aos verificados no estudo de Ayyanar e Subash-Babu (2012) de 0,70 \% para o jambolão in natura. Santos (2011) assegurou que a proteína contida nas hortaliças e frutas pode variar de 1 a $3 \%$, valor esse que concorda com o encontrado na PJAM.

A polpa de jambolão liofilizada (PJAM) apresentou valor alto para o teor de fibras totais se comparado ao corante de jambolão (CJAM) (Tabela 1). Shelke et al. (2020) incorporou o pó de bagaço de jambolão em sorvete e obteve que devido à presença de fibras no pó $(8,02 \%)$, houve uma redução na taxa de derretimento do sorvete, pois as fibras atuaram aumentando a capacidade de retenção de água.

De acordo com Panghal et al.( 2019), o emprego da polpa de jambolão em massa, resultou em um aumento do teor de fibra alimentar em $15,1 \%$, e em caso de aplicação em massas o alto teor de fibra pode causar um enfraquecimento na rede de glúten, também há uma relação direta com o índice de absorção de água e índice de solubilidade de água. Com a obtenção do corante natural é possível à redução do teor de fibra, consequentemente no caso da aplicação em massas alimentícias haverá uma facilidade na absorção de água, consequentemente um rápido cozimento, se comparado ao emprego da polpa de jambolão liofilizada.

Os teores de fibra encontrados para PJAM e CJAM indicam que o jambolão possui, além de seus compostos bioativos, características desejáveis de fibras para inclusão em novos produtos alimentícios.

A polpa de jambolão liofilizada (57,8 g.g-1) em comparação com o corante natural de jambolão (78,1 g.g-1) exibiu baixo teor de carboidratos (Tabela 1), o alto teor de carboidrato no CJAM é devido à adição da farinha de arroz, pois de acordo com Fitriani, Permana e Setiaboma (2019) a farinha de arroz possui 80,95\% de carboidrato, sendo o componente majoritário do material.

Os valores médios encontrados para o $\mathrm{pH}$ (Tabela 2) indicam que a PJAM e CJAM tem características de frutas cítricas ( $\mathrm{pH}$ abaixo de 4,5). Os valores médio de $\mathrm{pH}$ encontrados neste trabalho foi inferior ao relatado por Tavares et al. (2017) para polpa liofilizada de jambolão $(4,26)$ e superior $(3,64)$ ao encontrado por Kapoor, Ranote e Sharma (2014), $\mathrm{pH}$ 3.35 . 
O corante obteve um $\mathrm{pH}$ menor $(3,2)$ se comparado a polpa de jambolão liofilizada $(3,65)$, a farinha de arroz é adicionada com o intuito de manter a estabilidade do corante natural, pois a adição de um polissacarídeo é capaz de proteger as antocianinas da degradação com pH acima de 3,2 (CORTEZ et al., 2017).

A acidez média encontrada para a PJAM (Tabela 2) foi 0,59 \% inferior aos valores detectados por Soares (2014) em análise de polpa de jambolão de diversas regiões da cidade de Goiânia, Goiás, que variaram de 0,49 a 0,69 g ácido cítrico $100 \mathrm{~g}^{-1}$, sugerindo que os frutos avaliados neste trabalho estavam mais maduros. A CJAM apresentou acidez 1,76 vezes menor que a PJAM.

Tabela 2 - Médias e desvio padrão das características físico-químicas (pH, acidez titulável (AT) (g ácido cítrico 100g-1), sólidos solúveis totais (SST) ( ${ }^{\circ}$ Brix) da polpa de jambolão liofilizada (PJAM) e do corante natural de jambolão CJAM).

\begin{tabular}{ccc}
\hline Parâmetros & PJAM & CJAM \\
\hline $\mathrm{pH}$ & $3,64 \pm 0,08$ & $3,2 \pm 0,02$ \\
$\mathrm{AT}$ & $0,23 \pm 0,02$ & $0,13 \pm 0,02$ \\
$\mathrm{SST}$ & $4,6 \pm 0,14$ & $1,5 \pm 0,09$ \\
\hline
\end{tabular}

Fonte: Autores.

A média encontrada para os sólidos solúveis na PJAM (Tabela 2) demostra ser baixo, segundo Chim (2008) ao valor utilizado para determinar o ponto ideal de colheita de frutos deve estar entre 8-10 ${ }^{\circ}$ Brix. Os valores de sólidos solúveis totais das frutas apresentados neste trabalho são muito inferiores aos 9,0 ${ }^{\circ}$ Brix relatados por Lago, Gomes e Silva (2006) em jambolão. Essa diferença pode ser em decorrência do clima na época de colheita, o qual pode ter influenciado na alta síntese de açúcares, o que é decorrente da fotossíntese da planta. O menor valor de SST obtido para o CJAM em relação à PJAM foi devido à proporção de polpa utilizada na farinha de arroz para o processamento do corante em pó.

Apesar dos valores de sólidos solúveis da polpa do jambolão e do corante de jambolão serem baixas, esses valores podem ser ampliados com concentração em baixas temperaturas, o que matéria a sua riqueza em compostos bioativos.

\section{Compostos bioativos e atividade antioxidante}

Observa-se no presente estudo altos valores de compostos bioativos para a polpa (Tabela 3). Tavares et al. (2016) encontraram valores superiores de antocianinas (270 mg $100 \mathrm{~g}^{-1}$, cianidina 3-glicosideo), se comparado a este trabalho. Segundo Montilla et al, (2011) o teor de antocianinas pode ser influenciado por fatores climáticos como temperatura e iluminação, onde, dificultam a comparação entre diferentes cultivos de uma mesma fruta, e ainda mais quando se deseja comparar frutas diferentes avaliadas em trabalhos realizados em diferentes regiões, em épocas climáticas diferentes. Uma grande redução foi observada no CJAM em relação à PJAM, essa diminuição pode estar relacionada com a temperatura de secagem do corante e também devido à adição de farinha de arroz. Patras (2010) relata que o processo de secagem pode provocar alterações indesejáveis na amostra quanto aos valores de antocianinas.

A quantidade de compostos fenólicos para a PJAM (Tabela 3) pode ser considerado alto, visto que em estudo

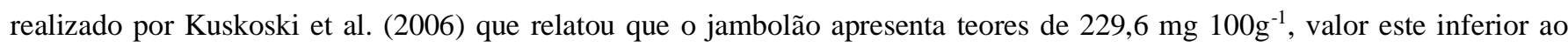
encontrado neste estudo. Outros frutos apresentaram valores superiores aos encontrados na PJAM liofilizada, como a amorapreta cultivar Tupy estudada por Chim (2008), a qual apresenta conteúdo de fenóis totais de 569,89 mg GAE.100g ${ }^{-1}$. Alguns trabalhos demonstram as propriedades antioxidantes dos compostos fenólicos e das antocianinas, significando que os alimentos 
ricos nestes compostos podem trazer benefícios à saúde. Segundo Rameshkumar et al. (2012) alguns fatores podem influenciar na quantidade de compostos fenólicos como clima, cultivar, solo e entre outros.

Os resultados demonstraram uma redução de 65 vezes dos fenólicos no CJAM em relação aos valores médios de PJAM. Parte dessa diminuição pode ser explicada pela utilização da farinha de arroz, e também devido à solubilidade dos compostos fenólicos, pois Barroca et al. (2013) relatam que a diminuição dos compostos fenólicos pode estar associada a solubilidade destes. O mesmo autor explica que essa degradação também pode ser atribuída à extensão de tempo e temperatura de secagem que o corante natural sofreu.

Tabela 3 - Médias e desvio padrão dos compostos bioativos, antocianinas (ANTO), compostos fenólicos (CF) e antioxidantes (ANT) da polpa de jambolão e corante natural.

\begin{tabular}{ccc}
\hline Parâmetros & PJAM & CJAM \\
\hline Antocianina $\left(\mathrm{mg} \cdot \mathrm{L}^{-1}\right)$ & $102,7 \pm 0,22$ & $17,18 \pm 0,31$ \\
Compostos fenólicos & $512,9 \pm 0,35$ & $180 \pm 0,33$ \\
$\left(\mathrm{mg} .100 \mathrm{~g}^{-1}\right)$ & $77,5 \pm 0,33$ & $6,40 \pm 0,30$ \\
\hline Capacidade Antioxidante $(\%)$ & & \\
\hline
\end{tabular}

Fonte: Autores.

A porcentagem de sequestro de radical livre de antioxidante (Tabela 3) apresentou um valor baixo para PJAM (77,5\%), se comparado ao encontrado por Kapoor, Ranote e Sharma, (2014) que obtiveram uma porcentagem de 88,34\%, porém a polpa de jambolão liofilizada teve uma baixa atividade antioxidante se comparado ao corante natural. Apesar de menores teores de antocianinas e compostos fenólicos, a farinha de arroz conseguiu proteger esses compostos.

A utilização de polpa de jambolão, devido aos seus compostos bioativos, tem sido estudada na incorporação de produtos alimentícios como sorvetes (Soares, 2018a), nectars (Soares, 2018a), fermentados alcoólicos (Oliveira, 2016), entre outros. Essa riqueza em compostos biotivos torna tanto a PJAM, quanto a CJAM, matérias-primas com potencial de utilização em novos produtos.

\section{Características tecnológicas}

Os resultados obtidos para a cor instrumental da polpa de jambolão (Tabela 4) indicam que essa possui coloração escura, tendendo para ao preto. Brito et al. (2017) caracterizou a polpa de jambolão como uma fruta de cor purpura-vermelha, devido a baixa luminosidade ( $\left.\mathrm{L}^{*} 22,20\right), \mathrm{C}^{*} 20,72, \mathrm{a}^{*} 20,30, \mathrm{~b}^{*}-4,17 \mathrm{e} \mathrm{h}^{\circ} 348,39$. Com o processo de secagem, no caso a liofilização, a coloração natural é alterada ou destruída durante o processamento ou armazenamento do produto (Oliveira et al., 2009).

O CJAM apresentou uma tendência ao branco $(72,3)$, devido à utilização da farinha de arroz no processamento do corante. A luminosidade da polpa liofilizada foi similar aos resultados relatados por Si et al. (2016), para pós de framboesa liofilizada $(35,26)$ e por Rocha (2013), para polpa de extrato de mirtilo $(24,55$ e 34,18), frutos também ricos em antocianinas.

Em relação à coordenada a* (verde ao vermelho), percebe-se que a PJAM e o CJAM (Tabela 4) apresentaram valores com tendência ao vermelho. Os valores encontrados para $\mathrm{a}^{*} \mathrm{e} \mathrm{b}^{*}$ apontam as tonalidades vermelha e azulada para as duas amostras. Augusta (2010) encontrou valores semelhantes para as coordenadas a* e b* em jambo vermelho em pó (19,07 e 9,94) respectivamente, aos encontrados neste estudo. A coordenada a* no CJAM $(13,9)$ foi menor se comparada ao PJAM $(24,3)$ essa variação é causada pela adição da farinha de arroz. 
O resultado obtido para pureza ou intensidade de cor c* (Tabela 4) apresentou maior saturação para PJAM $(25,2)$ e menor saturação e opacidade CJAM $(19,7)$, em relação ao ângulo hue $\left(h^{\circ}\right)$ o CJAM $(269,4)$ característica de cor purpura e PJAM $(309,7)$ uma tendência à cor vermelha.

Estudos demostram que a atividade de água tem sido considerada como atributo fundamental no controle de qualidade de um alimento (crescimento microbiológico e estabilidade). Para Chisté et al. (2006) 0,60 é o limite mínimo capaz de permitir o desenvolvimento de microrganismos em alimentos. Neste trabalho observa-se que tanto a PJAM quanto CJAM se apresentaram dentro dos limites mínimos relatados por Chisté et al. (2006).

De acordo com Santos et al. (2008), quando essas polpas de frutas são comercializadas secas o risco de contaminação diminui e retardam a rápida deterioração, que se limita principalmente a bolores e leveduras; sendo estes os mais importantes agentes de deterioração de polpas e sucos de frutas. Silva (2006) avaliando o teor de atividade de água em corante de urucum encontrou valores semelhantes $(0,62)$ aos relatados nesse estudo para CJAM.

Tabela 4 - Médias e desvio padrão das propriedades tecnológicas - cor ( $L^{*}, a^{*}, b^{*}, c^{*}$ e H), atividade de água (aw), índices de absorção em água (IAA), de solubilidade em água (ISA) e de absorção em óleo (IAO) da polpa de jambolão liofilizada (PJAM) e do corante natural de jambolão CJAM.

\begin{tabular}{ccc}
\hline Parâmetros & PJAM $^{\mathbf{1}}$ & CJAM $^{\mathbf{1}}$ \\
\hline L $^{*}$ & $34 \pm 0,45$ & $72,3 \pm 0,48$ \\
$\mathrm{a}^{*}$ & $24,3 \pm 0,41$ & $13,9 \pm 0,30$ \\
$\mathrm{~b}^{*}$ & $-7,2 \pm 0,41$ & $-14 \pm 0,27$ \\
$\mathrm{c}^{*}$ & $25,2 \pm 0,23$ & $19,7 \pm 0,22$ \\
$\mathrm{~h}^{\text {o }}$ & $309,7 \pm 0,01$ & $269,4 \pm 0,11$ \\
aw & $0,23 \pm 0,00$ & $0,55 \pm 0,00$ \\
IAA & $4,09 \pm 0,05$ & $1,60 \pm 0,02$ \\
IAO & $3,49 \pm 0,12$ & $2,18 \pm 0,06$ \\
ISA & $56,5 \pm 0,59$ & $10,3 \pm 0,31$ \\
\hline
\end{tabular}

Fonte: Autores.

Os resultados de IAA, ISA e IAO estão apresentados na Tabela 4. O índice de absorção de água de um alimento está relacionado à disponibilidade do grupo hidrofílico para se ligar as moléculas de água, formando um gel com as moléculas de amido (Alves et al., 2018), o PJAM $(4,09)$ apresentou a maior capacidade de absorção de água se comparado ao CJAM $(1,6)$, isto é devido ao maior teor de fibra presente no PJAM.

Observa-se que o ISA da PJAM (56,5) apresentou maior solubilidade se comparado ao CJAM (10,3), logo o CJAM absorve menos água e solubiliza menos que o PJAM. Matsuura (2005) determinou 25,9 \% de ISA para albedo de maracujá, valores abaixo aos encontrados neste trabalho para PJAM. Santana (2005) encontrou valores um pouco acima para as farinhas de laranja e maracujá desidratada por liofilizador $(57,7$ e 60,2), respectivamente. Essas diferenças se relacionam às características específicas de cada fruto.

O índice de absorção de óleo é importante, pois indicam as características de realçar sabor e melhorar sensação na boca, essenciais para elaboração de alimentos (Kaushal; Kumar; Sharma, 2012). Si et al. (2016) obtiveram para os pós de framboesa liofilizados um IAO de 2,10 $\mathrm{mL}_{\mathrm{g}} \mathrm{g}^{-1}$, valor semelhante ao encontrado para o CJAM $(2,18)$, enquanto para o PJAM apresentou um maior valor $(3,49)$. 
Entre os parâmetros tecnológicos avaliados, as coordenadas de cor são as que mais chamam a atenção tanto para a PJAM quanto para o CJAM. A cor acentuada do jambolão tem sido utilizada para valorizar alguns produtos (Soares et al., 2020), como que utilizam a própria fruta para sua produção ou até mistura com outras matérias-primas para inserir a cor intensa aos demais produtos.

\section{Propriedades térmicas e de pasta}

Os perfis viscoamilográficos, obtidos no RVA, da polpa de jambolão e corante natural de jambolão (Figura 1), mostram que o tempo de pico observado nas amostras variou de 25 a 500 segundos para a PJAM e 260 a 450 segundos para o corante natural. Segundo Lindeboo et al. (2005) o tempo de pico tem relação com o teor de amilose.

Figura 1 - Curvas RVA da polpa de jambolão (A) e corante de jambolão (B).
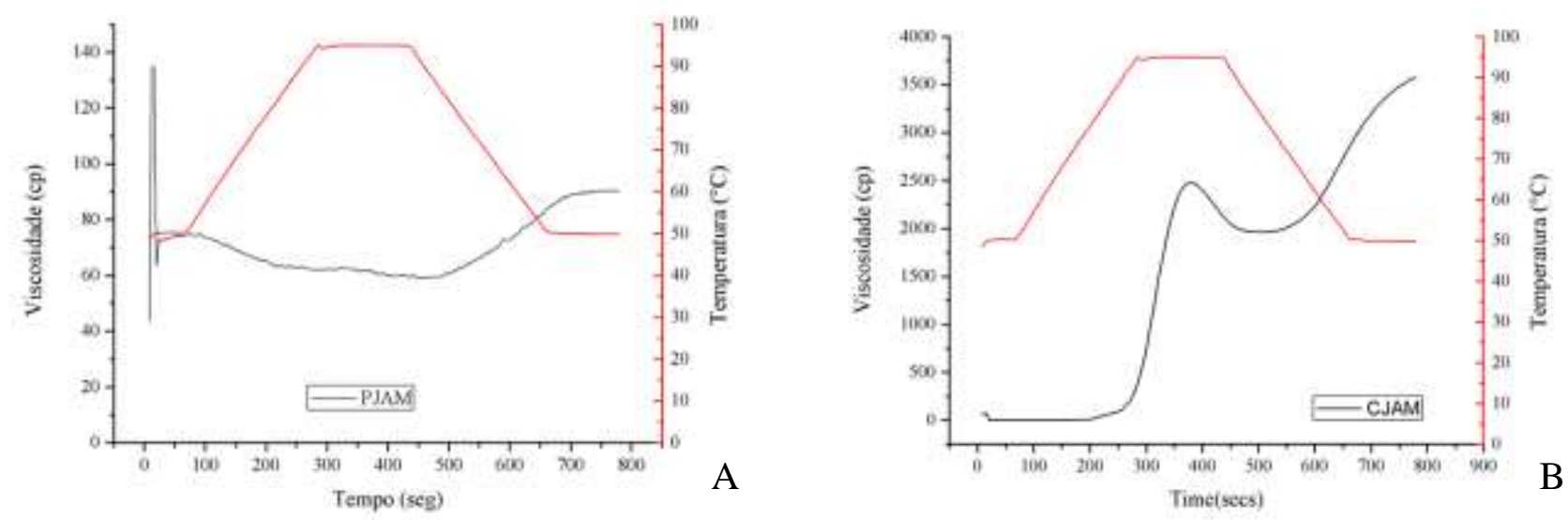

Fonte: Autores.

No presente estudo a temperatura de pasta para a PJAM $\left(48^{\circ} \mathrm{C}\right)$ e CJAM $\left(95,1{ }^{\circ} \mathrm{C}\right)$, assim a CJAM obteve maior temperatura de pasta, isto indica a presença de amido que é altamente resistente ao inchaço e ruptura (Kaushal; Kumar; Sharma, 2012). O CJAM também apresentou um pico de viscosidade alto (2038 cp), isto ocorreu devido à presença da farinha de arroz no processamento.

O PJAM $\left(48^{\circ} \mathrm{C}\right)$ apresentou a menor temperatura de pasta, isto corrobora com os resultados encontrados para índice de absorção de água, pois quanto menor a temperatura de pasta, maior será a capacidade de absorção de água (PrzetaczekRożnowska, 2017).

O conhecimento das curvas de viscosidade do PJAM e CJAM representam o comportamento durante o aquecimento desses produtos e permite avaliar as características da pasta formada, devido às modificações estruturais das moléculas, essa informação é importante para o desenvolvimento de novos produtos. 
Figura 2 - Curva do DSC da polpa de jambolão liofilizada.

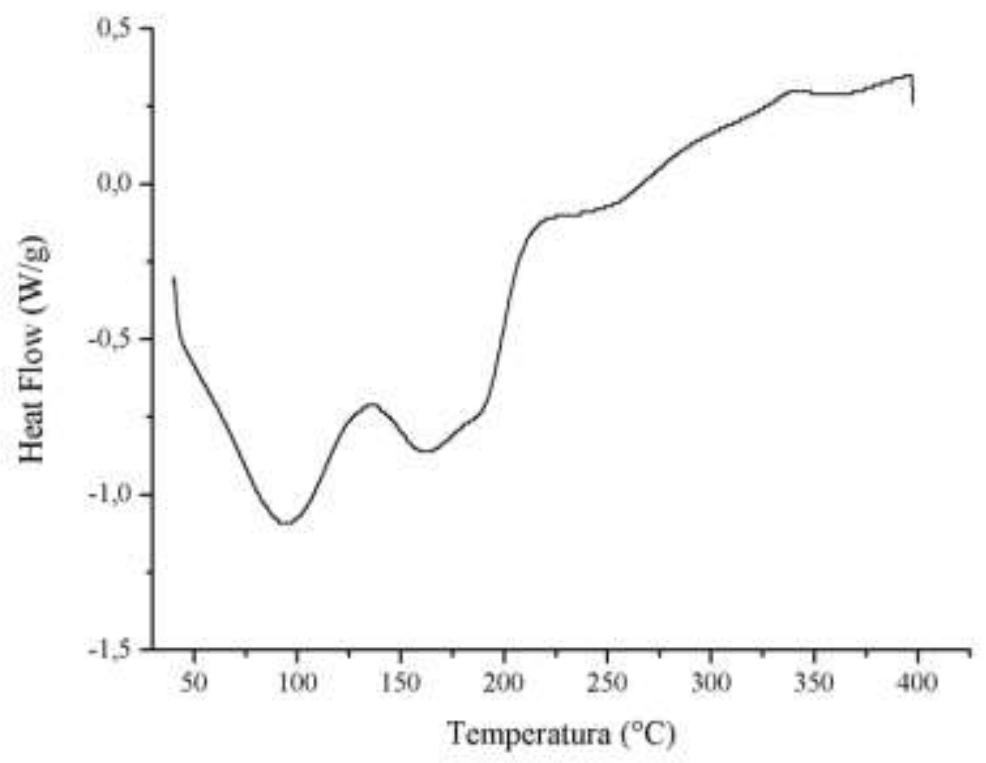

Fonte: Autores.

Na Figura 2 pode ser observar o estudo calorimétrico do corante natural de jambolão (CJAM) que foi obtido em uma faixa de temperatura de 50 a $400{ }^{\circ} \mathrm{C}$, com formação de três picos. Observa-se um pico exotérmico na temperatura de $140^{\circ} \mathrm{C}$, de acordo com Das, Goud e Das (2019) em seus estudos obtiveram o pó de extrato de antocianina de farelo de arroz roxo utilizando amido de arroz glutinoso como carreador, de acordo com os autores a formação do pico exotérmico $\left(157^{\circ} \mathrm{C}\right)$ ocorreu devido formação do complexo de cadeia longa de compostos amilose-fenólicos, pois estes necessitam de uma temperatura mais alta para se romper, assim é garantido um pó termicamente estável.

O conhecimento do comportamento térmico do CJAM indica a sua estabilidade em diferentes temperaturas.

\section{Microscopia eletrônica de varredura (MEV)}

Observa-se na Microscopia Eletrônica de Varredura (Figura 3 A, B, C) para polpa de jambolão que o processo de liofilização da polpa de jambolão mostrou formas irregulares e um aglomerado de partículas. Para Alves (2008), essas estruturas podem ser caracterizadas por uma condição desordenada em que não há repetições de forma geométrica e presença de faces planas bem estabelecidas, uma vez que essa característica é comum em produto liofilizado. 
Figura 3 - Imagens da microscopia eletrônica de varredura da polpa de jambolão (A, B, C), nos aumentos de 50, 350 e 3000x, e do corante de jambolão (D, E e F), nos aumentos de 50, 1500 e 2000x.
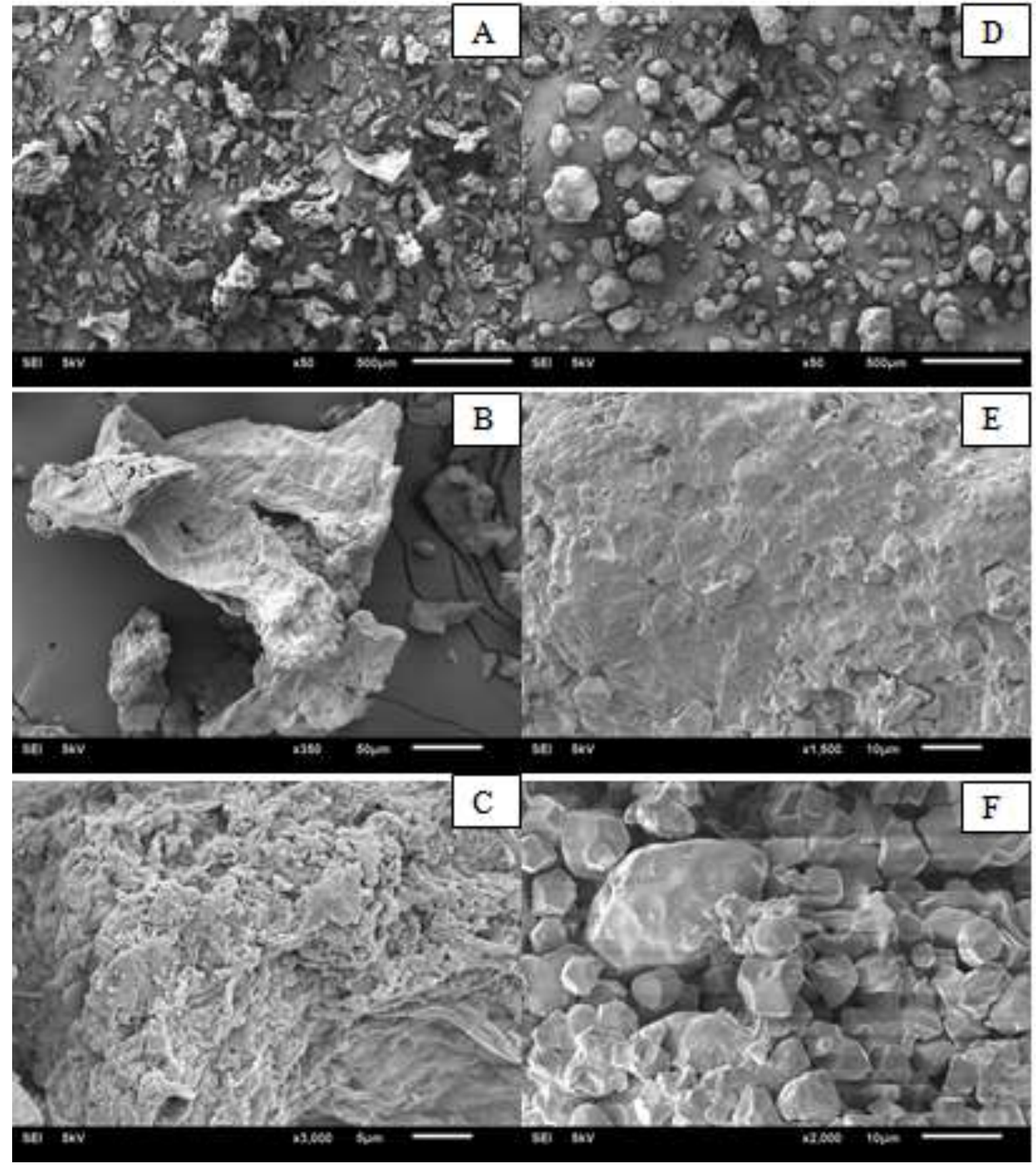

Fonte: Autores.

As imagens de MEV do corante de jambolão mostram na (Figura 3 D, E, F) partículas com aspecto angular e irregular, de tamanhos variados, demonstrando o aspecto do amido danificado, provavelmente durante a fabricação da farinha de arroz, acompanhado de partículas menores e irregulares que são partes da polpa liofilizada. Segundo Kaushik e Roos (2007); Fang e Bhandari (2010) a desuniformidade das partículas pode ser explicada pelo fato do corante ter sofrido uma secagem, além da diferença entre os tamanhos da polpa liofilizada e das partículas de farinha de arroz.

A microscopia eletrônica dos pós da PJAM e CJAM indicou uma variação em seus formatos. A polpa liofilizada apresentou, como esperado, muitas partículas de formatos irregulares que se relacionam com a característica fibrosa da fruta. O corante apresentou, além dos formatos irregulares originários da faz fibras, formatos do amido de arroz utilizado no processo 
de extração dos pigmentos.

\section{Conclusão}

Os resultados obtidos neste estudo permitiram concluir que: A matéria-prima utilizada para a extração do corante natural do jambolão é rica em fibra alimentar total e compostos bioativos, sendo então uma alternativa de aproveitamento desse fruto. É possível a utilização da polpa liofilizada do fruto de jambolão para o processo de extração de corantes naturais ricos em antocianinas e que o mesmo pode ser utilizado em alimentos naturais. Ocorreram perdas de compostos bioativos durante o processo de extração e secagem do corante de jambolão. O corante apresentou características para aplicação como espessante e aglutinante nos alimentos, devido sua propriedade de pasta. Os resultados obtidos, mesmo com a redução de alguns compostos, mostram que o jambolão é um alimento com propriedades antioxidantes e presença de bioativos, com potencial para ser utilizado como ingrediente funcional de alimentos. Sugere-se para trabalhos futuros a aplicação dos corantes de jambolão em diferentes alimentos, assim como o seu acompanhamento no decorrer do tempo.

\section{Referências}

AACC (American Association of Cereal Chemistry) (2000). AACC International approved methods of analysis. 10. th. St. Paul, MN, 2000. Approved November 8.

Alves, C. C. O., Resende, J. V., Cruvinel, R. S. R. \& Prado, M. E. T (2008). Estabilidade da microestrutura e do teor de carotenóides de pós obtidos da polpa de pequi (Caryocar brasiliense Camb.) liofilizada. Revista Ciência e Tecnologia de Alimentos, 28 (4), 830-839.

Alves, P. L. da S., Berrios, J. D. J., Pan, James \& Ascheri, J. L. R. (2018). Passion fruit shell flour and rice blends processed into fiber-rich expanded extrudates. CyTA - Journal of Food, 16 (1), 901-908.

Anderson, R. A., Conway, H. F., Pfeifer, V. F. \& Griffin Junior, E. L. (1969). Gelatinization of corn grits by Roll- and extrusion-cooking. Cereal Science Today, 14 (1), 4-12.

AOAC - Association of Official Analytical Chemists (1995). Official methods of analysis of the Association of the Analytical Chemists. (16th ed.), Washington.

AOAC - Association of Official Analytical Chemists (2006). Official methods of analysis of AOAC international. Food composition, additives, natural contaminants, (18 ed.), v.2, Gaithersburg: AOAC International.

AOAC - Association of Official Analytical Chemists (2010). Official methods of analysis of the Association of the Analytical Chemists. (18a ed.) $3^{\mathrm{a}}$ rev. Washington DC USA, p. 1096.

Augusta, I. M. Resende, J. M., Borges, S. V., Maia, M. C. A. \& Couto, A. P. G. (2010). Physical and chemical characterization of malay red-apple (Syzygium malaccensis, (L.) Merryl \& Perry) Skinandpulp. Ciências e Tecnologia de Alimentos, 30 (4), 928-932.

Ayyanar, M. \& Subash-Babu, P. (2012). Syzygium cumini (L.) Skeels: A review of its phytochemical constituents and traditional uses. Pac Journal Tropical Biomed, 2 (3), 240-246

Baraiya, N. S., Venkata, T., Rao, R. \& Thakkar, V. R. (2015). Improvement of Postharvest Quality and Storability of Jamun Fruit (Syzygium cumini L . var . Paras ) by Zein Coating Enriched with Antioxidants. Food and Bioprocess Technology, 8, 2225-2234.

Barroca, M. J., Guiné, R. P. F., Alves, M. A., Oliveira, S., Gonçalves, F. J.\& Correia, P. M. R. (2013). Effect of drying on the properties of pears cv. D. Joaquina. VII Congreso Iberico de Agroingeniería y Ciencias Hortícolas, Madrid, Spain.

Brito, B. de N. do C., Pena, R. da S., Lopes, A. S. \& Chisté, R. C. (2017). Anthocyanins of Jambolão (Syzygium cumini): Extraction and pH-Dependent Color Changes. Journal of Food Science, 82 (10), 2286-2290.

Carvalho, T. I. M. De, Lago-Vanzela, E. S., Rebello, L. P. G., Ramos, A. M., Gómez-Alonso, S., García-Romero, E., Da-Silva, R. \& Hermosín-Gutiérrez, I. (2016). Comprehensive study of the phenolic composition of the edible parts of jambolan fruit (Syzygium cumini (L.) Skeels). Food Research International, $82,1-13$.

Chim, J. F (2008). Caracterização de compostos bioativos em amora-preta (Rubussp.) e sua estabilidade no processo e armazenamento de geléias convencional e light. 87f. Tese (Doutorado em Ciência e Tecnologia Agroindustrial) - Universidade Federal de Pelotas, Pelotas-RS.

Chisté, R. C., Cohen, K. O., Mathias, E. A. \& Ramos Júnior, A. G. A (2006). Qualidade da farinha de mandioca do grupo seca. Ciência e Tecnologia de Alimentos, 26 (4), 861-864.

Cortez, R., Luna-Vital, D. A., Margulis, D. \& Gonzalez de Mejia, E. (2017). Natural Pigments: Stabilization Methods of Anthocyanins for Food Applications. Comprehensive Reviews in Food Science and Food Safety, 16 (1), 180-198.

Das, A. B., Goud, V. V. \& Das, C. (2019). Microencapsulation of anthocyanin extract from purple rice bran using modified rice starch and its effect on rice 
dough rheology. International Journal of Biological Macromolecules, 124, 573-581.

Fang, Z. \& Bhandari, B. (2010). Encapsulation of polyphenols: A Review. Trends in Food Science \& Technology, Cambridge, 21 (1), $510-523$.

Fitriani, V., Permana, L. \& Setiaboma, W. (2019). Chemical and Physical Charaterization of Cereal Flakes Formulated with Broken Rice and Banana Flour. IOP Conference Series: Earth and Environmental Science, 258 (1), 012003

Giusti, M. T. F. \& Wrolstad, R. (2003). Acylatedanthocyanins from edible sources and their applications in food systems: Review, Biochemical Engineering Journal, 14 (1), 217-225.

Haque, R., Sumiya, M. K., Sakib, N., Sarkar, O. S., Tarek, T., Siddique, I., Hossain, S., Islam, A. \& Parvez, A. K. (2017). Antimicrobial Activity of Jambul ( Syzygium cumini ) Fruit Extract on Enteric Pathogenic Bacteria. Advances in Microbiology, 07 (03), 195-204.

Kapoor, S., Ranote, S. \& Sharma, S. (2014). Bioactive Components and Quality Assessment of Jamun (Syzygium cumini L.) Powder Supplemented Chapatti. Indian Journal of Science and Technology, 8 (3), 287-295.

Kaushal, P., Kumar, V. \& Sharma, H. K. (2012). Comparative study of physicochemical, functional, antinutritional and pasting properties of taro (Colocasia esculenta), rice (Oryza sativa) flour, pigeonpea (Cajanus cajan) flour and their blends. LWT - Food Science and Technology, 48 (1), $59-68$.

Kaushik, V. \& Ross, Y. H (2007). Limonene encapsulation in freeze-drying of gum Arabic-sucrose-gelatin systems. LWT- Food Science and Technology, 40 (2), 1381-1391.

Kestemnbach H. \& Bota Fiilho W. J (1994). Microscopia Eletrônica de Transição e Varredura. Associação Brasileira de Metais.

Kuskoski, E. M., Asuero, A. G., Morales, M. T. \& Fett, R. (2006). Frutos tropicais silvestres e polpas de frutas congeladas: atividade antioxidante, polifenóis e antocianinas. Ciência Rural, 36 (4), 1283-1287.

Lago, E. S., Gomes, E. \& Silva, R. (2006). Produção de geléia de jambolão (Syzygium cumini Lamarck): processamento, parâmetros físico - químicos e avaliação sensorial. Ciência e Tecnologia de Alimentos, 26 (4), 847-852.

Larrauri, J. A., Rupérez, P. \& Saura-Calixto, F. (1997). Effect of Drying Temperature on the Stability of Polyphenols and Antioxidant Activity of Red Grape Pomace Peels. Journal of agricultural and food chemistry, 45 (4), 1390-1393.

Lestario, L. N., Howard, L. R., Brownmiller, C., Stebbins, N. B., Liyanage, R. \& Lay, J. O. (2017). Changes in polyphenolics during maturation of Java plum (Syzygium cumini Lam.). Food Research International, 100 (march), 385-391.

Lindeboom, D., Chang, P. R., Falk, K. C. \& Tyler, R. T. (2005). Characteristics of starch from eight quinoa lines. Cereal chemistry, 82 (2), $216-222$.

Lopes-Lutz, D., Alviano, D. S., Alviano, C. S. \& Kolodziejczyk, P. P. (2008). Screening of chemical composition, antimicrobial and antioxidant activities of Artemisia essential oils. Phytochemistry, 69 (8), 1732-1738.

Matsuura, F.C.A.U (2005). Estudo do albedo de maracujá e seu aproveitamento em barra de cereais. 138p. Tese (Doutor em Tecnologia de Alimentos) Faculdade de Engenharia de Alimentos. Universidade Estadual de Campinas, Campinas.

Montilla, E. C. Montilla, M. R., Arzaba, S., Hillebrand, P. \& Winterhalter. T. (2011). Anthocyanin composition of black carrot (Daucus carota ssp. Sativus var. atrorubens Alef.) cultivars Antonina, Beta Sweet, Deep Purple, and Purple Haze. Journal of Agricultural and Food Chemistry, 59 (3), 3385-3390.

Neacsu, M., Vaughan, N., Raikos, V., Multari, S., Duncan, G. J., Duthie, G. G. \& Russell, W. R. (2015). Phytochemical profile of commercially available food plant powders: Their potential role in healthier food reformulations. Food Chemistry, 179, 159-169.

Oliveira, A. C., Valentim, I. B., Goulart, M. O. F., Silva, C. A., Bechara, E. J. H. \& Trevisan, M. T. S. (2009). Fontes Vegetais Naturais de antioxidantes. Química Nova, 32, (3), 123-135.

Oliveira, V. B., Yamada, L. T., Fagg, C. W. \& Brandão, M. G. L. (2012). Native foods from Brazilian biodiversity as a source of bioactive biocomponds. Food Research International. Essex, 48 (10), 170-179.

Oliveira, E. R., Salgado, D. L., Santos, P. R., Soares Júnior, M.S., Vilas Boas, E. V. B., Caliari, M. (2017). Study of jambolan pulp bioactive compounds. Caderno de Ciências Agrárias, 9 (3), 54-59.

Oliveira, E. R., Caliari, M., Soares Júnior, M. S., Vilas Boas, E. V. B. (2016). Bioactive composition and sensory evaluation of blended jambolan (Syzygium cumini) and sugarcane alcoholic fermented beverages. Journal of the Institute of Brewing, 122, 719-728.

Panghal, A., Kaur, R., Janghu, S., SharmA, P., Sharma, P. \& Chhikara, N. (2019). Nutritional, phytochemical, functional and sensorial attributes of Syzygium cumini L. pulp incorporated pasta. Food Chemistry, 289, 723-728.

Patras, A. (2010). Effect of termal processig on anthocyanin stability in foods mechanisms and kinetics of degradation. Treands in Food Science \& Technology, 21 (1), 3-11.

Pereira, A. S., Shitsuka, D. M., Parreira, F. J., Shitsuka, R. (2018). Metodologia da pesquisa científica Santa Maria, RS: UFSM, NTE.

Prentice-Hernandez, C. \& Rusig, O. (1992). Extrato de urucum (Bixaorellana L.) obtido utilizando álcool etílico como solvente. Arquivo de Biologia e Tecnologia, 35 (1), 63-64.

Przetaczek-Rożnowska, I. (2017). Physicochemical properties of starches isolated from pumpkin compared with potato and corn starches. International Journal of Biological Macromolecules, 101, 536-542. 
Rameshkumar, A., Sivasudha, T., Jeyadevi, R., Ananth, D. A. \& Pradeepha, G. (2012). Effect of environmental factors [air and UV-C irradiation] on some fresh fruit juices, Food Research Technology, European, 234 (1), 1063-1070.

Rocha, C. T. (2013). Obtenção de suco misto de açaí, morango e acerola em pó por diferentes processos de secagem. Dissertação (Mestrado em Ciência e Tecnologia de Alimentos). Centro de Ciência Agrárias, Universidade Federal do Espírito Santo, ES.

Sabino, L. B. De S., Brito, E. S. de, Ivanildo, J. \& JU, S. (2018). Jambolan — Syzygium jambolanum. In: Exotic Fruits.

Santana, M. F. S. (2005). Caracterização físico-química de fibra alimentar de laranja e maracujá. 168p. Tese (Doutorado em engenharia de Alimentos), Faculdade de Engenharia de Alimentos, Universidade estadual de campinas, Campinas.

Santos, C. A. A., Coelho, A. F. S. \& Carreiro, S. C. (2008). Avaliação microbiológica de polpas de frutas congeladas. Ciência Tecnologia Alimentos, 4 (28), 913-915.

Santos, D. T. \& Meireles, M. A. A. (2011). Optimization of bioactive compounds extraction from jabuticaba (Myrciaria cauliflora) skins assisted by high pressure $\mathrm{CO}_{2}$. Innovative Food Science and Emerging Technologies, 12 (3), 398-406.

Shelke, G., Kad, V., Yenge, G., Desai, S., Kakde, S. (2020). Utilization of jamun pomace as functional ingredients to enhance the physico-chemical and sensory characteristics of ice cream. Journal of Food Processing and Preservation, 44 (10), e14736.

Si, X., Chen, Q., Bi, J., Wu, X., Yi, J., Zhou, L. \& Li, Z. (2016). Comparison of different drying methods on the physical properties, bioactive compounds and antioxidant activity of raspberry powders. Journal of the Science of Food and Agriculture, [S. l.], 96 (6), $2055-2062$.

Silva, M. L. A (2006). Extração de corantes de urucum (bixaorellana L.) utilizando sistema de recirculação de solventes. 92p. Dissertação (Mestrado Ciência e Tecnologia de Alimentos) - Departamento de Tecnologia de Alimentos, Universidade Federal do Pará.

Silva, W. P. da, Nunes, J. S., Gomes, J. P. \& silva, C. M. D. P. da S. (2018). Obtaining anthocyanin from jambolan fruit: kinetics, extraction rate, and prediction of process time for different agitation frequencies. Food Science \& Nutrition, 6 (6), 1664-1669.

Singh, B. \& Singh, J. P. (2018). Review Insights into the phenolic compounds present in jambolan (Syzygium cumini) along with their health-promoting effects. International Journal of Food Science and Technology, 53 (11), 2431-2447.

Singh, J. P, Kaur, A., Singh, N., NIM, L., Shevkani, K., Kaur, H. \& Arora, D. S. (2016). In vitro antioxidant and antimicrobial properties of jambolan (Syzygium cumini) fruit polyphenols. LWT - Food Science and Technology, 65 (1), 1025-1030.

Sniffen, C. J., O’Connor, J. D., Van Soest, P. J., Fox, D. G. \& Russell, J. B. (1992). A net carbohydrate and protein system for evaluating cattle diets: II. Carbohydrate and protein availability. Journal of animal science, 70 (11), 3562-3577.

Soares, N. M., Hornes, M. O., Perlin, F. S., pereira, D. T. \& Santos, M. (2014). Vinho de jambolão (Syzygium cumini) elaborado por duas formas de obtenção de polpa. Farroupilha (RS): Instituto Federal de Farroupilha, 5 p.

Soares, J. C, Garcia, M. C., Garcia, L. G. C., Caliari, M., Soares Junior, M. S. (2018). Jambolan sherbets overrun, color, and acceptance in relation to the sugar, milk, and pulp contents in formulation. Food Science and Technology, 38 (suppl.1), 313-318.

Soares, J. C, Soares Junior, M. S., Ferreira, K. C., Caliari, M. (2019). Physicochemical characteristics and sensory acceptance of jambolan nectars (Syzygium cumini). Food Science and Technology, 39 (suppl.1), 8-14.

Soares, J. C. , Soares Junior, M. S., Numa, I. A. N. , Pastore, G. M., Caliari, M. (2020). Jambolan nectar: physical and chemical properties due to formulation ingredients. Research, Society and Development, 9 (5), e161953212.

Tavares, I. M. C., Nogueira, T. Y. K., Mauro, M. A., Gómez-Alonso, S., Gomes, E., Da-Silva, R., Hermosín-Gutiérrez, I. \& Lago-Vanzela, E. S. (2017). Dehydration of jambolan [Syzygium cumini ( L .)] juice during foam mat drying: Quantitative and qualitative changes of the phenolic compounds. Food Research International, 102 (june), 32-42. 